\title{
An experimental study of boundary-layer transition induced vibrations on a hydrofoil
}

\author{
Antoine Ducoin ${ }^{\mathrm{a}, *}$, Jacques André Astolfi ${ }^{\mathrm{b}}$, Marie-Laure Gobert ${ }^{\mathrm{c}}$ \\ a Department of Naval Architecture and Marine Engineering, University of Michigan, Ann Arbor 48104, USA \\ ${ }^{\mathrm{b}}$ Institut de Recherche de l'Ecole Navale EA 3634, Ecole Navale, 29240 Brest Cedex 9, France \\ ${ }^{\mathrm{c}}$ ENI Val de Loire, Université François Rabelais de Tours, LMR Laboratory, 3 rue de la chocolaterie, BP 3410, F-41034 Blois Cedex, France
}

Keywords:

Vibrations

Laminar Separation Bubble

Fluid structure interaction

\begin{abstract}
A B S T R A C T
This paper aims at characterizing experimentally laminar to turbulent transition induced vibrations. Here, the transition is known to be triggered by a Laminar Separation Bubble that results from a laminar separation of the boundary-layer flow on a hydrofoil. In this study we consider two NACA66312 (Mod) laminar hydrofoils at low angles of incidence (mostly $2^{\circ}$ and $4^{\circ}$ ) and Reynolds numbers ranging from $R e=450000$ to 1200000 , in order to get transitional regimes. The first hydrofoil, made of steel $\left(E=2.1 \times 10^{11} \mathrm{~Pa}\right)$, is referred to as the rigid hydrofoil, although it is seen to vibrate under the action of the LSB. To better understand the possible interaction between the flow and the foil vibrations, vibration measurements are repeated using a flexible hydrofoil $\left(E=3 \times 10^{9} \mathrm{~Pa}\right)$ of same geometry (under zero loading) and in close configurations. The experiments are carried out at the French Naval Academy Research Institute (IRENav, France). Wall pressure and flow velocity measurements enable a characterization of the laminar separation bubble and the identification of a vortex shedding at a given frequency. It is hence shown that the boundary-layer transition induces important foil vibrations, whose characteristics in terms of frequency and amplitude depend on the vortex shedding frequency, and can be coupled with natural frequencies of the hydrofoils.
\end{abstract}

\section{Introduction}

The hydrodynamic loading prediction plays an important role in the marine structure design of lifting bodies. In some cases, a good knowledge of the boundary-layer flow regime around the body is required. For instance, the control surfaces of autonomous underwater vehicles (AUV) can operate at transitional regimes due to their relatively small scales. The appearance of the transition in the boundary-layer flow may affect their hydrodynamic performances and be responsible for the generation of vibrations. Typically, the transition on lifting bodies occurs at low to moderate Reynolds numbers and is triggered by a separated flow region due to an adverse pressure gradient. The development of the turbulent flow, which causes a momentum transfer in the wall normal direction, allows the flow to reattach. This phenomenon can also appear at higher Reynolds numbers on laminar sections, designed in the marine industry for the propeller blades, which promote a laminar boundary-layer flow along their chords. However, because of the large Reynolds number, a very strong and located vorticity region may occur and affect the pressure distribution.

\footnotetext{
* Corresponding author.

E-mail addresses: aducoin@umich.edu, antoine.ducoin@laposte.net (A. Ducoin).
} 
Many studies have been dedicated to the issue of laminar separation, in particular for aerodynamic applications on flat plates and lifting bodies. The Laminar Separation Bubble (LSB) on foils at moderate Reynolds numbers has been given a special interest, from the pioneering experimental works of Gaster (1966) or Tani (1964) to recent numerical studies involving DNS. It has hence been shown that the transitional region appears near the trailing edge for low to moderate angles of incidence, and moves towards the leading edge as the angle of incidence increases, and that the bubble length is inversely related to the adverse pressure gradient. DNS simulations performed by Alam and Sandham (2000) showed that complex and multi-scaled structures can occur in the LSB wake. Moreover, the presence of vortex sheddings resulting from a flapping motion of the bubble was highlighted in some recent DNS studies; Rist and Maucher (2002), for instance, found that in the case of a flat plate the vortex sheddings generate characteristic velocity and pressure fluctuations downstream of the bubble. Pauley et al. (1990) studied an unsteady Laminar Separation Bubble induced by an adverse pressure gradient on a leading edge geometry. They found that the frequency of the vortex shedding from the bubble could be made dimensionless using a Strouhal number $\mathrm{St}_{\theta}$ based on the momentum thickness $(\theta)_{\text {sep }}$ and the external velocity $\left(v_{\text {ext }}\right)_{\text {sep }}$ at laminar separation. This Strouhal number was used in recent works to characterize the LSB, see Yang and Voke (2001) and Marquillie and Ehrenstein (2003) for instance. An innovative experimental study of Hain et al. (2009) uses a time-resolved particle image velocimetry (TR-PIV) system to determine the dominant frequencies of the transition process and the flapping of the bubble along an airfoil. The latter has a SD7003 section and the flow is characterized by a Reynolds number of $R e=66000$. A high accurate description in time of the shedding process is presented. Although the measured vortex shedding frequency proved to be multi-component, it was possible to identify dominant structures which led to large magnitudes in the frequency spectrum. Recently, Jones et al. (2008) performed a numerical analysis of a LSB in the case of an airfoil at $\alpha=5^{\circ}$ and a low Reynolds number $\operatorname{Re}_{c}=50000$. The transition to turbulence was analyzed in terms of vorticity and wall pressure fluctuations. It appears that the vorticity grows significantly downstream of the LSB location, like in Rist and Maucher (2002) on a flat plate. At the same time, periodic and intermittent pressure fluctuations are found in the near-LSB region, induced by coherent vortex sheddings. Far away from the LSB region near the trailing edge, the vorticity increases in a thick boundary-layer and the pressure fluctuations become random, which is characteristic of turbulent fluctuations. Later on, Almutairi et al. (2010) investigated the bursting of Laminar Separation Bubble on the same hydrofoil near stall and found a good agreement with the previous DNS simulations and experiments. In that case, it appears that the onset of the Laminar Separation Bubble plays a role in the stall for high angles of incidence, also observed by Richez et al. (2008). Ducoin et al. (2009) investigated the wall pressure fluctuations along a NACA66 hydrofoil in the case of a transient pitching motion from $0^{\circ}$ to $15^{\circ}$ at $\mathrm{Re}=750000$. They showed that the transition together with the vortex shedding of the LSB creates large-amplitude periodic pressure fluctuations downstream of the bubble. The frequency of the LSB vortex shedding was seen to remain constant as the angle of attack varies, even if higher frequency components were observed.

In the case of a flexible foil, such fluctuations are likely to induce important vibrations, that may in return affect the boundary-layer flow. Poirel et al. (2008) studied the self-sustained oscillations of a NACA0012 airfoil. The low to moderate Reynolds numbers considered suggest that the Laminar Separation Bubble plays an important role in the foil oscillation. Moreover, the laminar to turbulent transition was seen to strongly affect the lift coefficient. This case has been studied later for limit cycle oscillation, see Poirel and Yuan (2010). Such interactions have also been studied through experimental and numerical approaches for membrane airfoils. This case has been studied later for limit cycle oscillation, see Poirel and Yuan (2010). Lian and Shyy (2007), for instance, performed a numerical simulation for $\mathrm{Re}=60000$ of a hydrofoil partly covered with a latex membrane, which was seen to vibrate under the pressure fluctuations generated by the LSB. The presence of this vibrating portion affected the positions of the separation and of the transition, compared to the case of an entirely rigid airfoil in the same conditions, even though the lift and drag mean coefficients were close to the rigid hydrofoil values. From their numerical simulations involving a latex-membrane airfoil, Visbal et al. (2009) showed that for a low Reynolds number $\operatorname{Re}=2500$, the presence of the fluid led into a mean camber of the airfoil, as well as large membrane fluctuations for high angles of incidence. The vibrations proved to affect the boundary-layer flow at separation, resulting in improved aerodynamic performances. At a higher Reynolds number $\mathrm{Re}=48500$, the boundary-layer was seen to become rapidly turbulent from the leading edge, and the resulting multi-scaled coherent flow structures induced more regular but smaller vibrations. Such results were retrieved by the numerical investigations of Gordnier (2009) who obtained a strong correlation between the frequencies arising from the membrane airfoil vibration and the vortex shedding frequencies in the boundary-layer flow.

The aim of the present paper is to investigate, through an experimental study, the vibratory response of a flexible hydrofoil subjected to a transitional regime induced by a Laminar Separation Bubble. The Reynolds number ranges from $\operatorname{Re}=450000$ to $\operatorname{Re}=1200000$ and is relatively high compared to previous related studies. However, due to the laminar section and the low angles of attack considered $\left(\alpha=2^{\circ}\right.$ and $\left.\alpha=4^{\circ}\right)$, the critical values for the transition onset are such that the chosen Reynolds numbers should induce a transition along the chord. Flow velocity and wall pressure measurements are first carried out on a steel hydrofoil, at given angle of incidence $2^{\circ}$ and Reynolds number 750 000. Additional angles of incidence are also considered for the pressure measurements. The results provide qualitative and quantitative characterizations of the LSB and the following laminar to turbulent transition. Despite its high stiffness, the latter hydrofoil, which will be referred to as the rigid hydrofoil in the following, was seen to vibrate under the transitional boundary-layer flow. Vibration measurements are then presented for various angles of incidence and Reynolds numbers. The frequency associated with the transition is identified and its effects on the hydrofoil vibrations are discussed. In order to enhance the observed phenomena, further vibration measurements are performed on a flexible hydrofoil in close configurations. The results are analyzed in the light of previous rigid hydrofoil results, in terms of LSB characteristics and vibrations, and help to assess the influence of the LSB and transition process on the hydrofoil vibrations. 


\section{Experimental set-up}

Measurements are carried out in the cavitation tunnel at IRENav. The test section is $1 \mathrm{~m}$ long and has a square section of length $h=0.192 \mathrm{~m}$. The facility provides velocities ranging from 0 to $15 \mathrm{~m} / \mathrm{s}$, and pressures from $30 \mathrm{mbar}$ to 3 bars. The tunnel turbulence intensity defined as $V_{\mathrm{rms}} / V$ at the center of the test-section is about $2 \%$. It is a medium turbulence intensity close to the lower limit of turbulence intensity of large pipes or hydrodynamic tunnels, ranging generally between $1 \%$ and $5 \%$. Both rigid and flexible hydrofoils are NACA 66-shaped, and present a camber type NACA $a=0.8$, a camber ratio of $2 \%$ and a relative thickness of $12 \%$ (Leroux et al., 2005). They are mounted horizontally in the tunnel test section (Fig. 1). Their chords $c=0.150 \mathrm{~m}$ and spans $b=0.191 \mathrm{~m}$ correspond to a low aspect ratio $b / c=1.3$ and a confinement parameter $h / c=1.28$. The hydrofoils can rotate, providing a large range of angles of incidence for the hydrofoils. In the following experiments we will consider angles of incidence between $\alpha=2^{\circ}$ and $\alpha=8^{\circ}$. The rigid hydrofoil is made of stainless steel $\left(E=2.1 \times 10^{11} \mathrm{~Pa}\right)$. It is partially cantilevered using a steel cylinder connected to a motor which is used to change the angle of attack (see Fig. 1); and the tip section is free. The flexible hydrofoil is made of polyacetate ( $\mathrm{POM}, E=3 \times 10^{9} \mathrm{~Pa}$ ) chosen in order to significantly increase the level of deformations and vibrations. Unlike the rigid hydrofoil, it is fully clamped at the root, whereas the tip section is free.

Different freestream velocities ranging from 3 to $8 \mathrm{~m} / \mathrm{s}$ have been considered in our experiments. The flow is hence characterized by Reynolds numbers based on the foil chord length between 450000 and 1200000 .

Pressure measurements are carried out using seventeen piezo-resistive transducers (Keller AG 2 MI PAA100-075-010) of 10 bars maximum pressure. The pressure transducers are mounted into small cavities with a $0.5 \mathrm{~mm}$ diameter pinhole at the rigid hydrofoil surface. The wall pressure spectra measured by the transducers are attenuated from the theoretical cut-off frequency $f_{c}=9152 \mathrm{~Hz}$. Experiments are led with a sample frequency of $f=20 \mathrm{kHz}$.

The transducers locations are given in Fig. 1. As shown, a set of ten transducers is aligned along the chord on the suction side at mid-span, starting from the leading edge at reduced coordinate $x / c=0.1$ up to the trailing edge at coordinate $x / c=0.9$, with a step of $0.1 c$. In this paper we will focus on the transducers located in the LSB region near the trailing edge, that is to say from $x / c=0.6$ to $x / c=0.9$. Two sets of three transducers are arranged parallel to this line in order to analyze three-dimensional effects (which are, however, beyond the scope of this paper).

Velocity measurements have been carried out for a nominal freestream velocity $V_{\infty}$ is $5 \mathrm{~m} / \mathrm{s}$, corresponding to a Reynolds number $\operatorname{Re}=750000$. The velocities are measured on the suction side of the foil using a DANTEC two component LDA system, which provides a $0.5 \mathrm{~mm}$ long ( $Z$ direction) and $0.04 \mathrm{~mm}$ wide ( $X$ and $Y$ directions) measurement volume. It was coupled with two DANTEC enhanced Burst Spectrum Analyzers and a DANTEC Burstware software. The origin $(X=0$, $Y=0$ ) of the positioning system is set at the leading edge of the foil at zero angle of incidence. Measurements are performed in the vicinity of the pressure measurements. The laser beams were aligned with the spanwise direction allowing us to approach closely the surface foil. In our case, only longitudinal velocities $u$ are measured due to the near wall measurements into the boundary-layer which is below $1 \mathrm{~mm}$ high. Therefore, one laser signal is consistently lost. At each measurement location, the resulting velocity corresponds to the time-averaged value over the measurement length.

Velocities are mapped along normal lines to the foil surface. The measurement positions along the normals are selected with a logarithmic progression in order to obtain an accurate resolution of the velocity field close to the foil surface, as shown in Fig. 2 for $\alpha=2^{\circ}$. The distribution of the normals along the chord is set according to the knowledge of the LSB location for $\alpha=2^{\circ}$, which has been previously studied using wall pressure transducers (see Ducoin et al., 2009). Therefore, the mesh is refined near

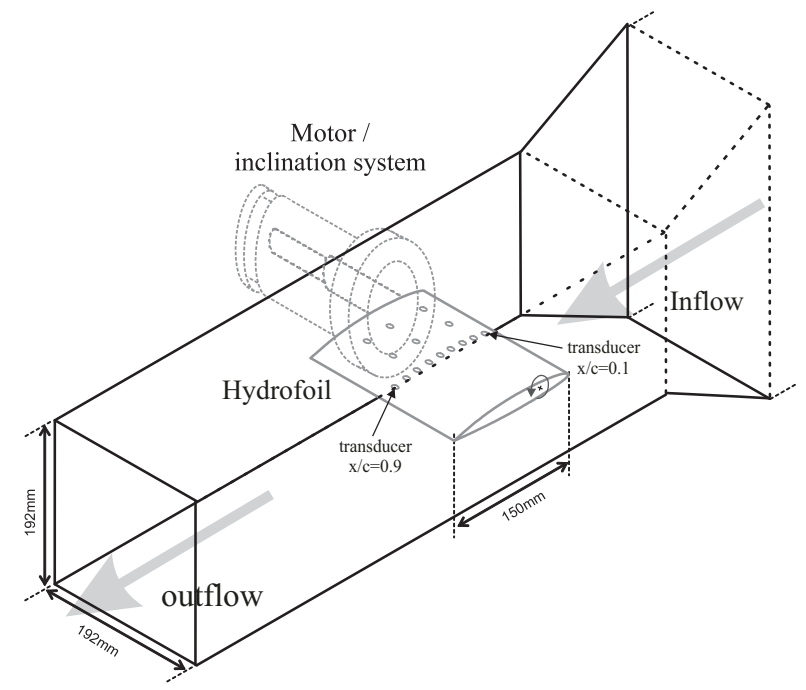

Fig. 1. Experimental set-up, rigid hydrofoil. 


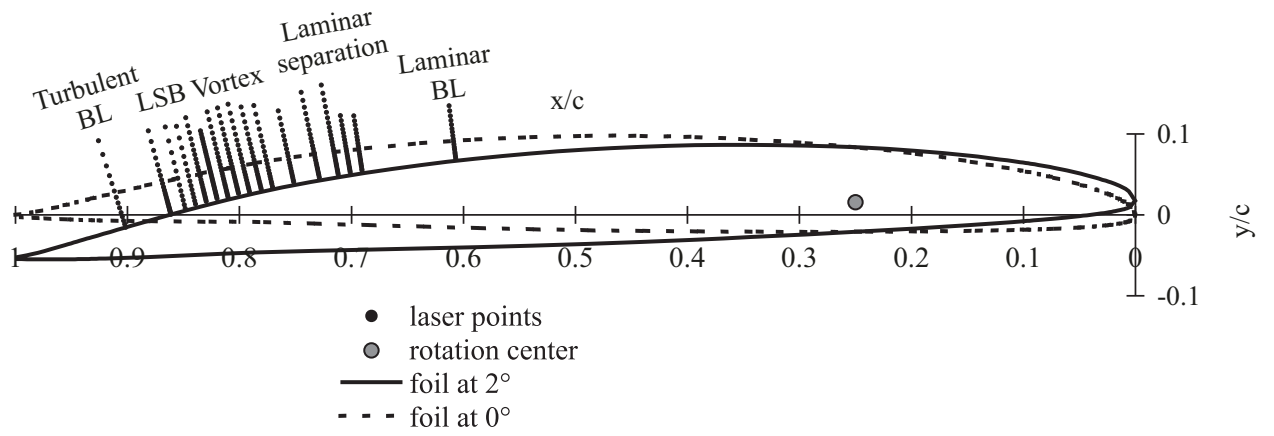

Fig. 2. LDV measurement locations along normals to the hydrofoil surface for $\alpha=2^{\circ}$.

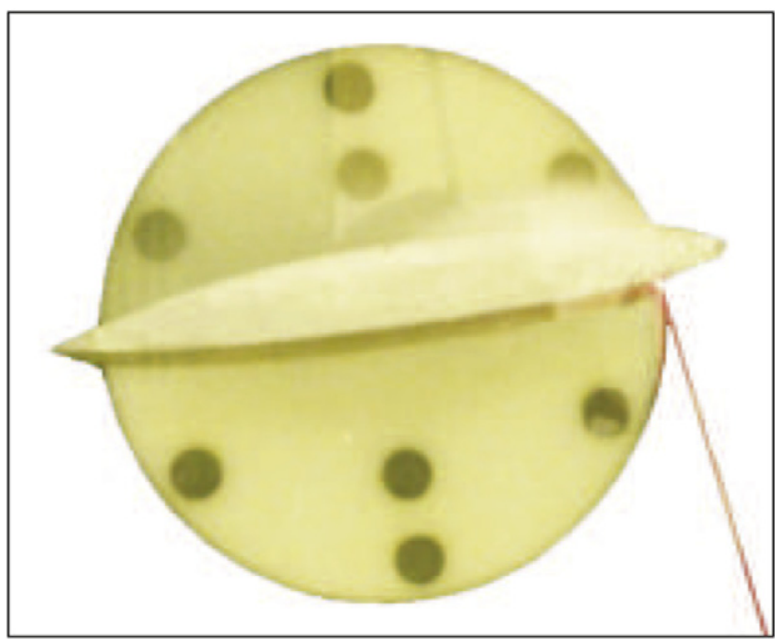

Fig. 3. Laser vibrometry measurements on the pressure side (the straight line is the laser beam), flexible hydrofoil.

the separation point around $x / c=0.69$, as well as around the LSB vortex and the turbulent reattachment positions, where transitional boundary-layer and high pressure fluctuations occur, that is to say between $x / c=0.76$ and $x / c=0.84$.

The normal lines are fitted in order to have the first measured point at a $y=0 \mathrm{~mm}$ coordinate from the foil surface. The random uncertainty associated with the electronic and numerical treatment of the Doppler signal giving the instantaneous velocity is estimated to be less than $1.5 \%$. The uncertainty of the absolute location in the normal direction with respect to the foil surface was of about one tenth of millimeter. Although the error appears small, it implies that a relative uncertainty still exists for the location of measurement points in the close vicinity of the foil surface.

Structural vibrations are measured by means of a Laser Doppler Vibrometer Polytec PSV-400, using a class II He-Ne laser of wavelength $\lambda=633 \mathrm{~nm}$. The device is equipped with a scanning system that enables vibration measurements over a predefined grid mapped on the vibrating surface. The measure is optimized for a plane surface subjected to vibrations in its normal direction, the normal velocities being captured with sensitivities ranging from 10 to $1000 \mathrm{~mm} / \mathrm{s} / \mathrm{V}$. At each measurement point of the grid, the software provides a Fourier transform of the velocity signal, possibly averaged from a user-defined number of acquisitions, and for frequencies up to $40 \mathrm{kHz}$. In the following, sets of 32-64 acquisitions will be considered to compute the spectra. Moreover, the preservation of the phase information throughout the scan, thanks to an appropriate reference signal, is useful to retrieve the operating deflection shape at a given frequency, and therefore to identify the natural frequencies of the structure, and their associated vibration modes, from the individual or grid-averaged velocity spectra.

The measurements of the hydrofoils vibrations are performed on their pressure sides through the transparent bottom wall of the tunnel test section, the vibrometer scanning head being fixed on the ground. Low-frequency vibrations related to the tunnel structure may hence been detected along with the foil vibrations during the experiment. Considering the geometry of the tunnel test section, the scanning head has to be inclined from its vertical position, the laser beam hence having an angle of incidence with the hydrofoil chord from about $45^{\circ}$ near the trailing edge to $90^{\circ}$ near the leading edge, as shown in Fig. 3 .

To improve the accuracy of the results, several single-point vibration measurements have been carried out in addition to scanning measurements over the hydrofoils pressure sides. In this configuration, the number of acquisitions used to 
compute the velocity spectra has been increased, and in the case of the flexible hydrofoil, reflective patches have been stuck on the measurement locations to avoid the light-absorption effect due to the hydrofoil material.

\section{Results and discussion}

\subsection{Velocity measurements}

Boundary-layer velocity measurements are carried out for the angle of incidence of $2^{\circ}$ around the transitional region. The results are summarized in Fig. 4 which shows the velocity profiles along the chord from $x / c=0.6$ to $x / c=0.9$. The scenario expected in the case of a transition from laminar to turbulent induced by a LSB is well described. At $x / c=0.6$, the boundary-layer is thin and laminar. The laminar separation is found at about $x / c=0.72$, then the velocity profiles are disturbed near the wall, as for instance at $x / c=0.79$, due to reverse flow. Downstream of this region, a transitional boundary-layer is observed (Fig. 4 at $x / c=0.84$ ) and the flow is turbulent at about $x / c=0.9$.

The different stages of the LSB-induced transition are illustrated from the velocity profiles in Figs. 5-7. Fig. 5 first details the velocity profiles measured between $x / c=0.6$ and $x / c=0.74$. The boundary-layer is seen laminar, with a thickness less

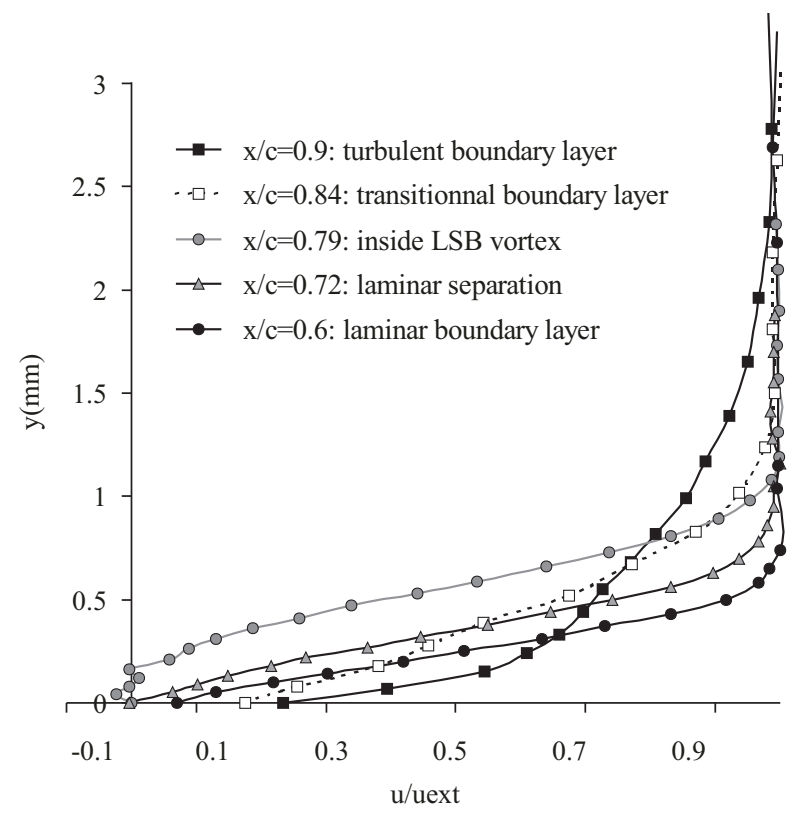

Fig. 4. Mean velocity profiles from $x / c=0.6$ to $x / c=0.9$ for $\alpha=2^{\circ}$ and $\operatorname{Re}=750000$.

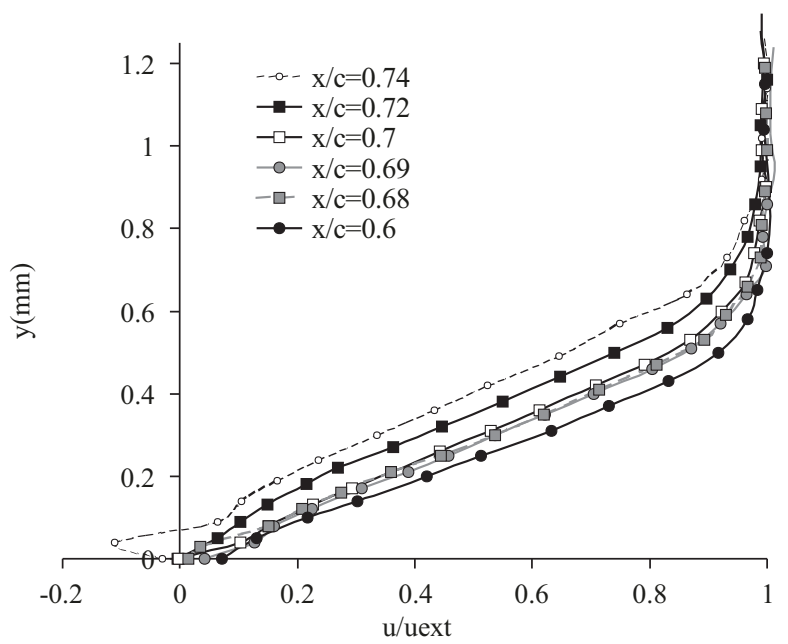

Fig. 5. Mean velocity profiles at laminar separation $\left(\alpha=2^{\circ}, \operatorname{Re}=750000\right)$. 


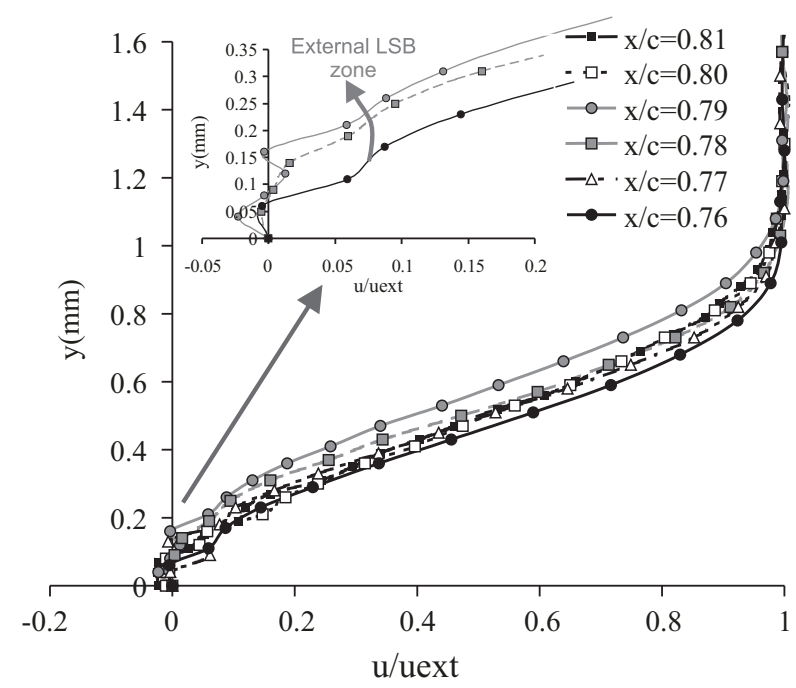

Fig. 6. Mean velocity profiles in the LSB, vortex region and turbulent reattachment $\left(\alpha=2^{\circ}, \operatorname{Re}=750000\right)$.

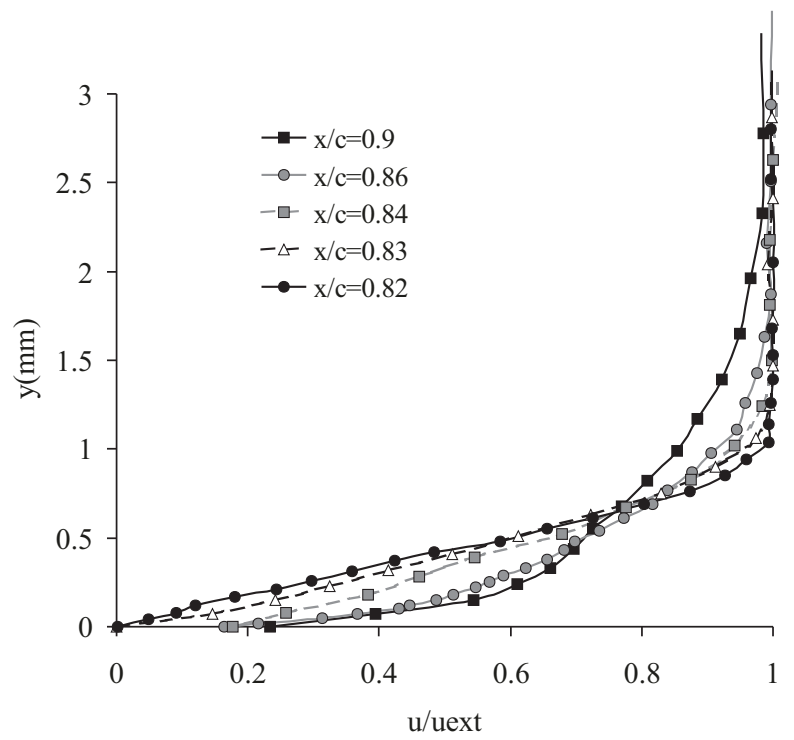

Fig. 7. Mean velocity profiles around the LSB, transition to turbulence $\left(\alpha=2^{\circ}, \operatorname{Re}=750000\right)$.

than $1 \mathrm{~mm}$. The thinnest boundary-layer is located at $x / c=0.6$, then the thickness increases as the location gets closer to the trailing edge. Between $x / c=0.72$ and $x / c=0.74$, the velocity profiles show an inflection which is identified as the laminar separation.

The next velocity profiles, measured between $x / c=0.76$ and $x / c=0.81$ into the vortex region of the LSB, are shown in Fig. 6. In the close vicinity of the hydrofoil surface, the density of particles needed by the LDV system to estimate the flow velocity decreases, which may induce precision errors in the values of the measured velocities. In this region, negative and positive velocity values that are expected to occur are not clearly detected. However, the velocity profiles of Fig. 6 clearly exhibit a series of near-zero values close to the surface, which reveal the presence of reversed flow characteristic of the LSB vortex region. Moreover, we observe that the boundary-layer thickness increases up to $x / c=0.79$ and then decreases up to $x / c=0.81$. The LSB thickness, which is characterized by a velocity drop in the transition region between the LSB and the external flow, (see zoom in Fig. 6), follows the same evolution. Therefore, the LSB maximum thickness is found for $x / c=0.79$ and is about 0.2 to $0.25 \mathrm{~mm}$.

Fig. 7 illustrates the scenario of transition to turbulence. The boundary-layer is seen transitional from $x / c=0.82$ to $x / c=0.84$, with a thickness of about 1 to $1.2 \mathrm{~mm}$, and then becomes turbulent from $x / c=0.86$. The maximum thickness observed on Fig. 7 is located at $x / c=0.9$ and is about $2.2 \mathrm{~mm}$. 
To complete the analysis, the measured values of the longitudinal velocity component $u$ have been gathered and interpolated over the measurement grid (see Fig. 2). Fig. 8 represents the resulting contour map of the dimensionless velocity $u / u_{\text {ext }}$ (with $u_{\text {ext }}$ the external velocity to the boundary-layer). This shape of a Laminar Separation Bubble is well known, see for example Haggmark et al. (2000). The presence of the LSB, characterized by negative to small positive values, can be easily spotted between $x / c \approx 0.7$ and $x / c \approx 0.82$. The growth of the boundary-layer thickness downstream of the bubble, which reveals the establishment of a turbulent regime, is also clearly visible. The turbulent reattachment point toward the back of the LSB may then be located around $x / c=0.83$, which gives a LSB length of about $10 \%$ of the chord.

Table 1 details the boundary-layer characteristics along the suction side of the hydrofoil for $\alpha=2^{\circ}$, deduced from the velocity measurements.

The boundary-layer is characterized by the shape coefficient defined as $H=\delta^{*} / \theta$, with $\delta^{*}$ the boundary-layer displacement thickness and $\theta$ the boundary-layer momentum thickness. To compute the latter two thicknesses, the limit of integration is chosen such that the difference of longitudinal velocities between two consecutive points in the $y$ direction is less than $1 \%$. The shape coefficient is useful to localize the laminar separation, where $H$ reaches a value of 3.5 , and to discriminate the laminar boundary-layer, for which $H=2.6$, from the turbulent one, characterized by $H=1.6$ (according to previous numerical studies). First, the results obtained at $x / c=0.6$, where $H=2.43$, meets the expected value for a laminar boundary-layer. The laminar separation may then be located between $x / c=0.72$ and $x / c=0.74$, where $H$ ranges between 3.379 and 3.959. The LSB region is then observed between $x / c=0.76$ and $x / c=0.81$ and is characterized by $H$ values larger than 3.5. At $x / c=0.83$, the shape factor reaches the value of $H \approx 2$ characteristic of a transitional boundary-layer, and then a turbulent value $H=1.61$ is retrieved at $x / c=0.86$.

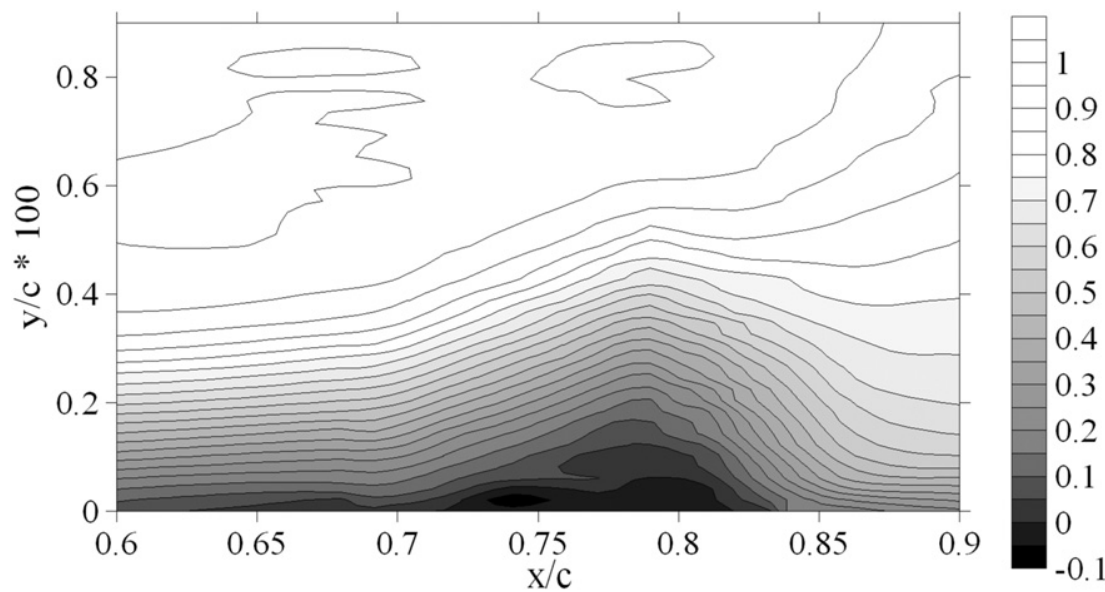

Fig. 8. Laminar Separation Bubble near the trailing edge: isovalues of the longitudinal velocity component $u / u_{\text {ext }}$ through the boundary-layer for $\alpha=2^{\circ}$ and $\operatorname{Re}=750000$.

Table 1

Boundary-layer characteristics for $\alpha=2^{\circ}$ and $\operatorname{Re}=750000$.

\begin{tabular}{|c|c|c|c|}
\hline Location on chord & $\delta^{*}(\mathrm{~mm})$ & $\theta(\mathrm{mm})$ & $H$ \\
\hline 0.6 & 0.230 & 0.095 & 2.43 \\
\hline 0.68 & 0.317 & 0.104 & 3.049 \\
\hline 0.69 & 0.307 & 0.108 & 2.852 \\
\hline 0.7 & 0.338 & 0.108 & 3.119 \\
\hline 0.72 & 0.379 & 0.112 & 3.379 \\
\hline 0.74 & 0.447 & 0.113 & 3.959 \\
\hline 0.76 & 0.499 & 0.123 & 4.050 \\
\hline 0.77 & 0.534 & 0.127 & 4.198 \\
\hline 0.78 & 0.564 & 0.124 & 4.548 \\
\hline 0.79 & 0.544 & 0.124 & 4.383 \\
\hline 0.8 & 0.466 & 0.128 & 3.619 \\
\hline 0.81 & 0.529 & 0.132 & 4.025 \\
\hline 0.82 & 0.472 & 0.171 & 2.757 \\
\hline 0.83 & 0.382 & 0.167 & 2.286 \\
\hline 0.84 & 0.395 & 0.185 & 2.139 \\
\hline 0.86 & 0.403 & 0.250 & 1.61 \\
\hline 0.9 & 0.457 & 0.287 & 1.593 \\
\hline
\end{tabular}


Given the decreasing data rate values at the vicinity of the hydrofoil surface, spectral analyses of velocity fluctuations have not been considered in the present study. However, valuable information can be obtained from spectral analyses of wall pressure measurements, as shown in the next section.

\subsection{Wall pressure measurements}

Wall pressure measurements were carried out for the rigid hydrofoil at $\alpha=2^{\circ}$ during a period of $T=1 \mathrm{~s}$. The results are shown in Fig. 9 for three locations $x / c=0.7, x / c=0.8$ and $x / c=0.9$. Very low amplitude and random fluctuations due to noise are observed at $x / c=0.7$, Fig. 9(a). Assuming that the laminar separation is located between $x / c=0.72$ and $x / c=0.74$, the pressure transducer therefore lies in the laminar and unperturbed boundary-layer. At $x / c=0.8$ (Fig. 9(b)), high amplitude
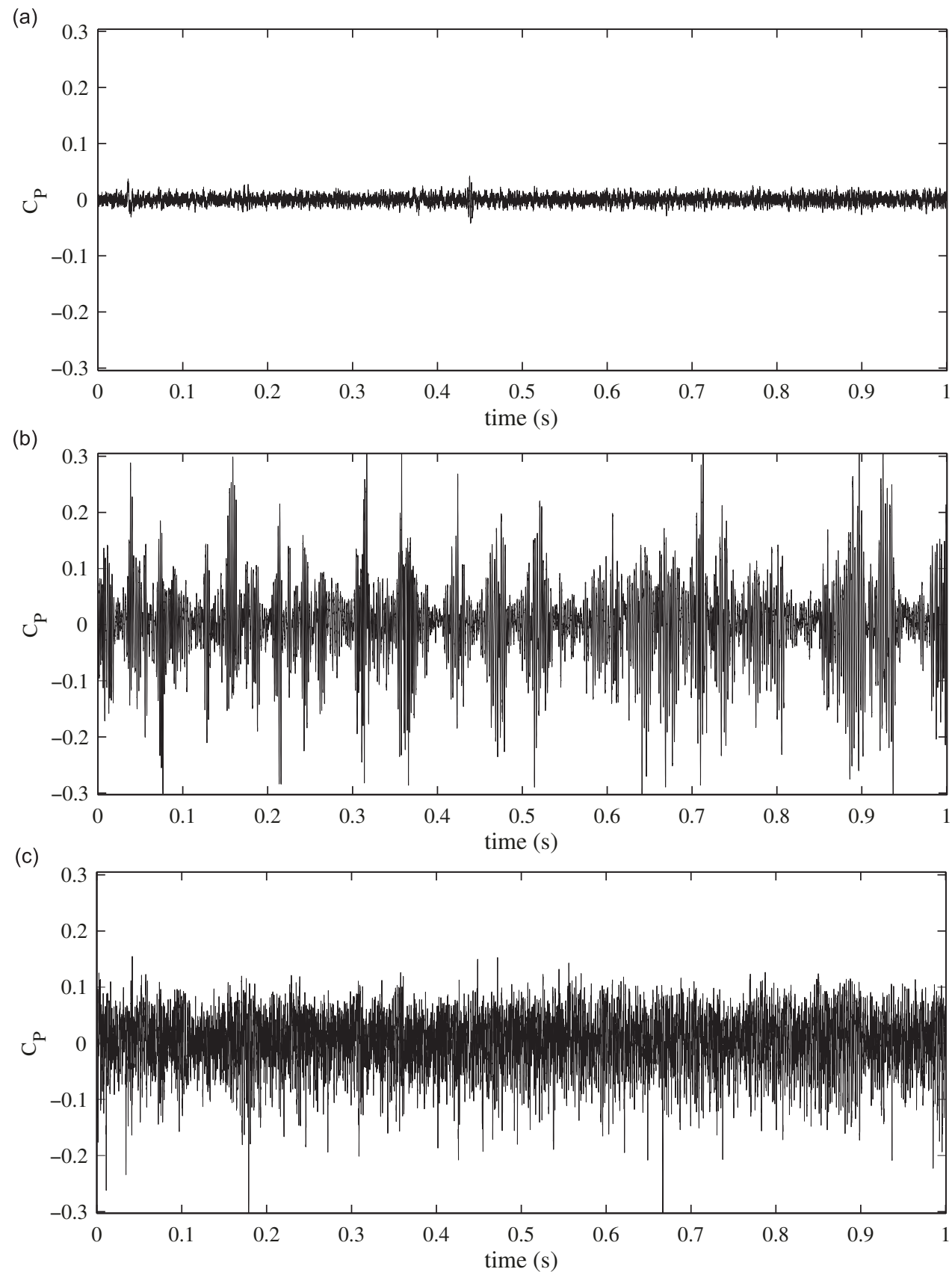

Fig. 9. Wall pressure fluctuations for an angle of incidence $\alpha=2^{\circ}$ and various transducers along the suction side, $\operatorname{Re}=750000$ : (a) $x / c=0.7$, (b) $x / c=0.8$, and $(\mathrm{c}) x / c=0.9$. 
fluctuations of about $\Delta C_{P}=0.3$ are observed, characterized by an intermittent behaviour. Moreover, these fluctuations seem periodic and are located under the reverse-flow vortex towards the back of the LSB (see Fig. 6), where the boundary-layer is highly disturbed. Then, at $x / c=0.9$ (Fig. 9 (c)), the level of fluctuations decreases $\left(\Delta C_{P} \approx 0.1\right)$ and gets denser within an approximately constant envelope, confirming the turbulent behaviour of the boundary-layer at this location.

The pressure signal fluctuations are post-processed using spectrograms to highlight possible characteristic frequencies. The spectrograms are short-time frequency spectra computed from signal segments of 256 points, that is, considering the sampling frequency $f=20 \mathrm{kHz}$, of length $T=0.0128 \mathrm{~s}$. For each time-segment, a Fast Fourier Transform is computed using a Hamming window, with a precision $\Delta f=4.88 \mathrm{~Hz}$. An overlap of $50 \%$ (that is, 128 points) is chosen between the segments, and the frequency spectra are hence estimated every $0.0064 \mathrm{~s}$. The spectrograms of the two fluctuating pressure signals, corresponding to $x / c=0.8$ and $x / c=0.9$, are depicted in Fig. 10. For $x / c=0.8$ (Fig. 10(a)), the spectrogram presents highamplitude frequency peaks, relatively localized between a main frequency $f \approx 400 \mathrm{~Hz}$, and a secondary frequency about $600 \mathrm{~Hz}$.

These fluctuations are clearly not turbulent, but the consequence of a vortex shedding process with an intermittent behaviour. Downstream of this location, the fluctuations appear to be more random, as a result of the turbulence development (Fig. 10b)). It may be noticed that the main frequency of the vortex shedding $f \approx 400 \mathrm{~Hz}$ is still visible, however, with a lower amplitude.

Further Fourier transform computations of the wall pressure fluctuations have been performed to get a more precise value of the specific frequencies mentioned above. For this purpose, Fast Fourier Transforms is computed for the transducer location $x / c=0.8$, using pressure signal recorded over a period of length $T=4 \mathrm{~s}$ with a time step $\Delta T=5 \times 10^{-4} \mathrm{~s}$. The precision of the Fourier transform is therefore $\Delta f=0.25 \mathrm{~Hz}$. The resulting average frequency spectra is shown in Fig. 11. The trends raised from the analysis of the previous spectrograms are retrieved, and the dominating vortex shedding frequency may be estimated as $f=395 \mathrm{~Hz}$, whereas the secondary frequency is about $576 \mathrm{~Hz}$. As shown, the spectrum does not exhibit specific low-frequency components, however, a closer examination unveiled the existence of two peaks on both sides of the main frequency, shifted by $\pm 22 \mathrm{~Hz}$ and hence at 373 and $417 \mathrm{~Hz}$, respectively. They have to be linked to the low-frequency modulation clearly visible in Fig. 9 (b). At $x / c=0.8$, the pressure signal is therefore typically an Amplitude Modulated signal, characterized by a high-frequency signal at $395 \mathrm{~Hz}$ related to the vortex shedding, and modulated in amplitude by a low-frequency signal of $22 \mathrm{~Hz}$ that reveals a low-frequency modulation originating from the LSB. However, these observations do not allow to raise definitive conclusions regarding the origin of the secondary peak at $575 \mathrm{~Hz}$. It could be related to a higher frequency component appearing during transition. More experimental data is therefore needed to investigate this issue, which could be the subject of future works.

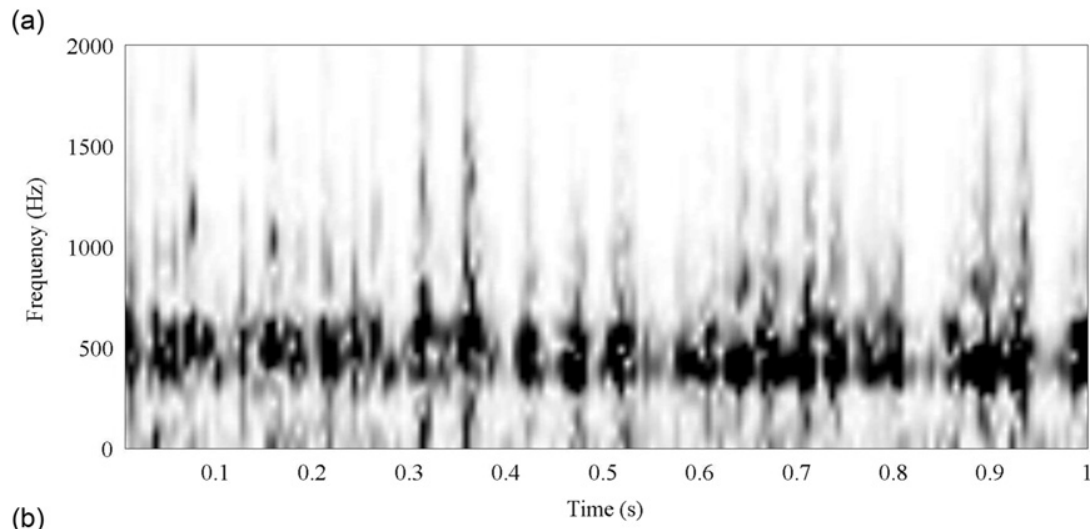

(b)

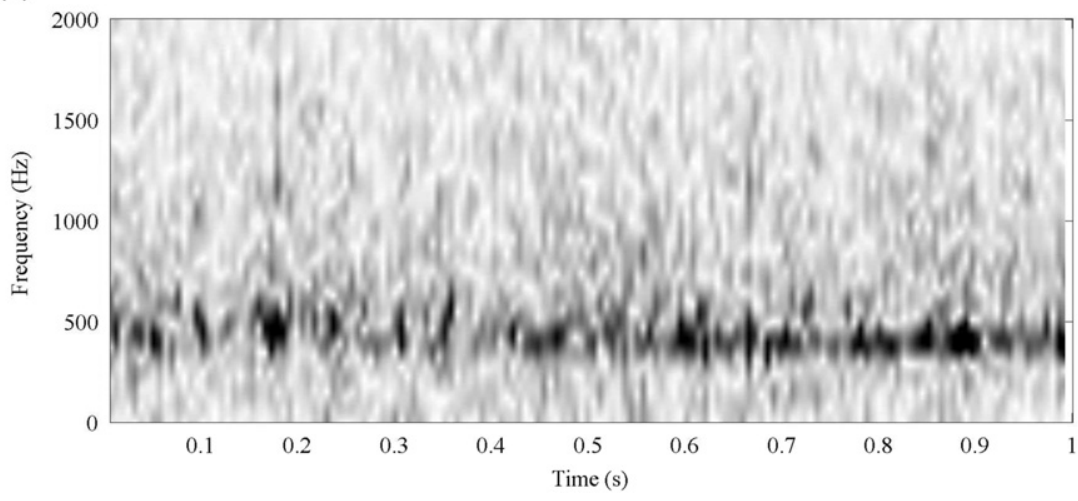

Fig. 10. Spectrograms of wall pressure fluctuations at $\alpha=2^{\circ}$ for two transducers along the suction side, $\operatorname{Re}=750000$ : (a) $x / c=0.8$ and (b) $x / c=0.9$. 


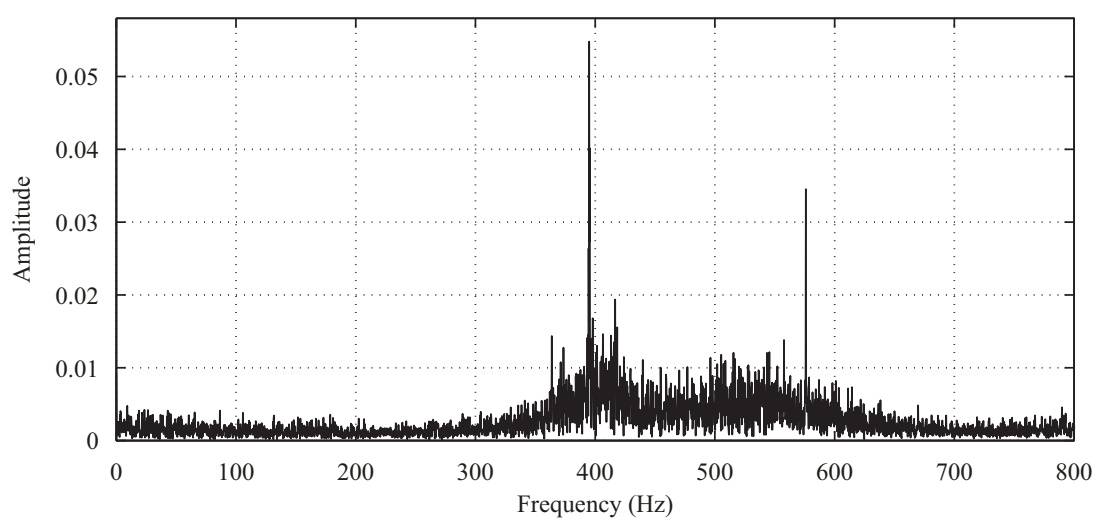

Fig. 11. Frequency spectra of the wall pressure fluctuations at $x / c=0.8$ for $\alpha=2^{\circ}$ and $\operatorname{Re}=750000$.
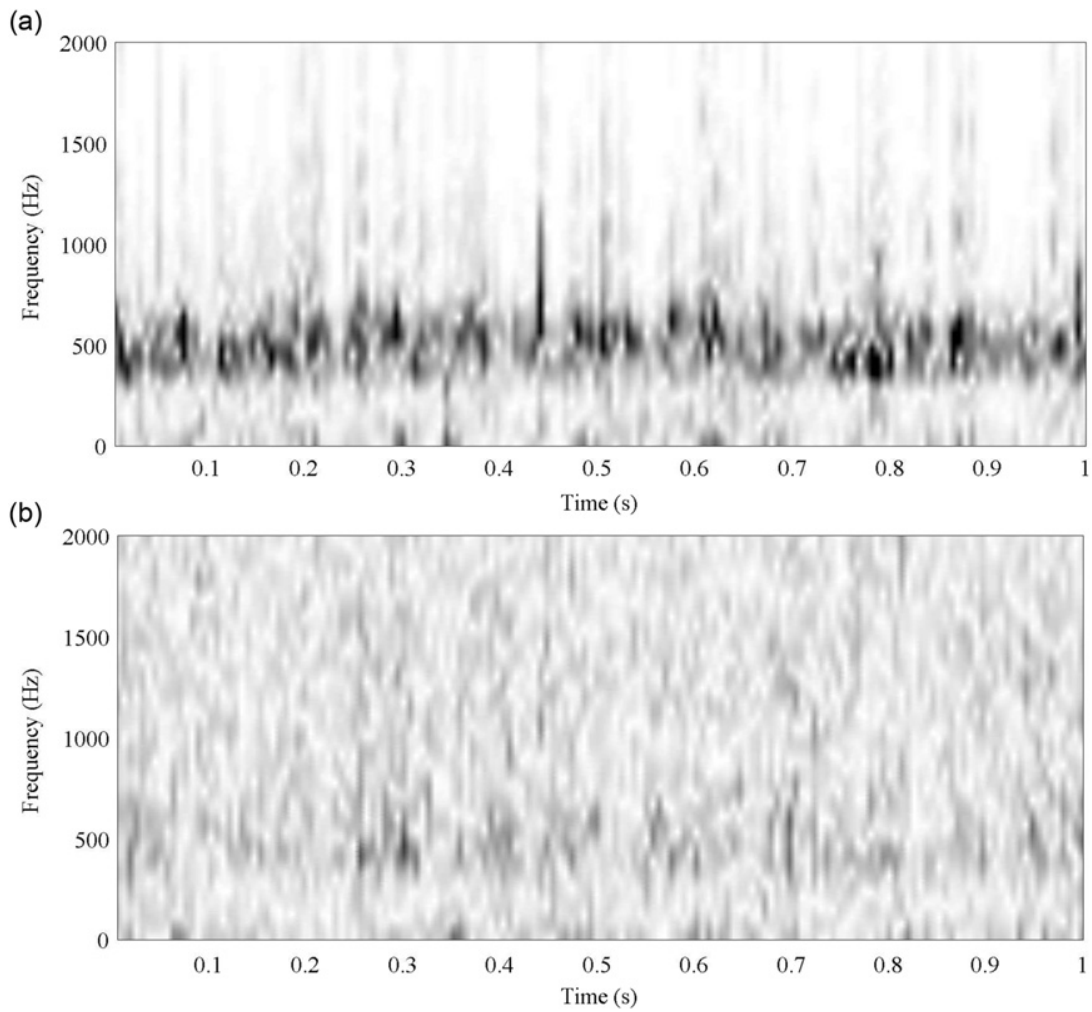

Fig. 12. Spectrograms of wall pressure fluctuations at $\alpha=4^{\circ}$ for two transducers along the suction side, $\operatorname{Re}=750000$ : (a) $x / c=0.7$ and (b) $x / c=0.8$.

Fig. 12 displays wall pressure spectrograms at a higher angle of incidence $\alpha=4^{\circ}$. Comparing those results to the previous ones at $\alpha=2^{\circ}$, we observe that the highest level of periodic fluctuations has moved from $x / c=0.8$ to $x / c=0.7$, due to the development of the pressure gradient from the leading edge. This result confirms the expected displacement of the Laminar Separation Bubble toward the leading edge of the hydrofoil. The dominant frequency, clearly visible at $x / c=0.7$, is unchanged and remains around $f=400 \mathrm{~Hz}$. This observation is consistent with the fact that although the LSB comes closer to the leading edge as the angle of incidence increases, the boundary-layer thickness is very little modified in this region, hence the LSB keeps overall the same characteristics (see Ducoin et al., 2009). At $x / c=0.8$, the fluctuations are here more random and the flow is almost fully turbulent. Again, the main frequency of pressure fluctuations may, however, still be observed at a lower amplitude.

Measurements performed at higher angles of incidence $\alpha \geq 5^{\circ}$ revealed that the transition is in these cases located at the leading edge of the hydrofoil, and that the boundary-layer is fully turbulent over the whole suction side. Spectrograms, obtained at $x / c=0.7$ and $x / c=0.8$, are computed for $\alpha=6^{\circ}$. Fig. 13 shows that the spectrum changes in a broadband 

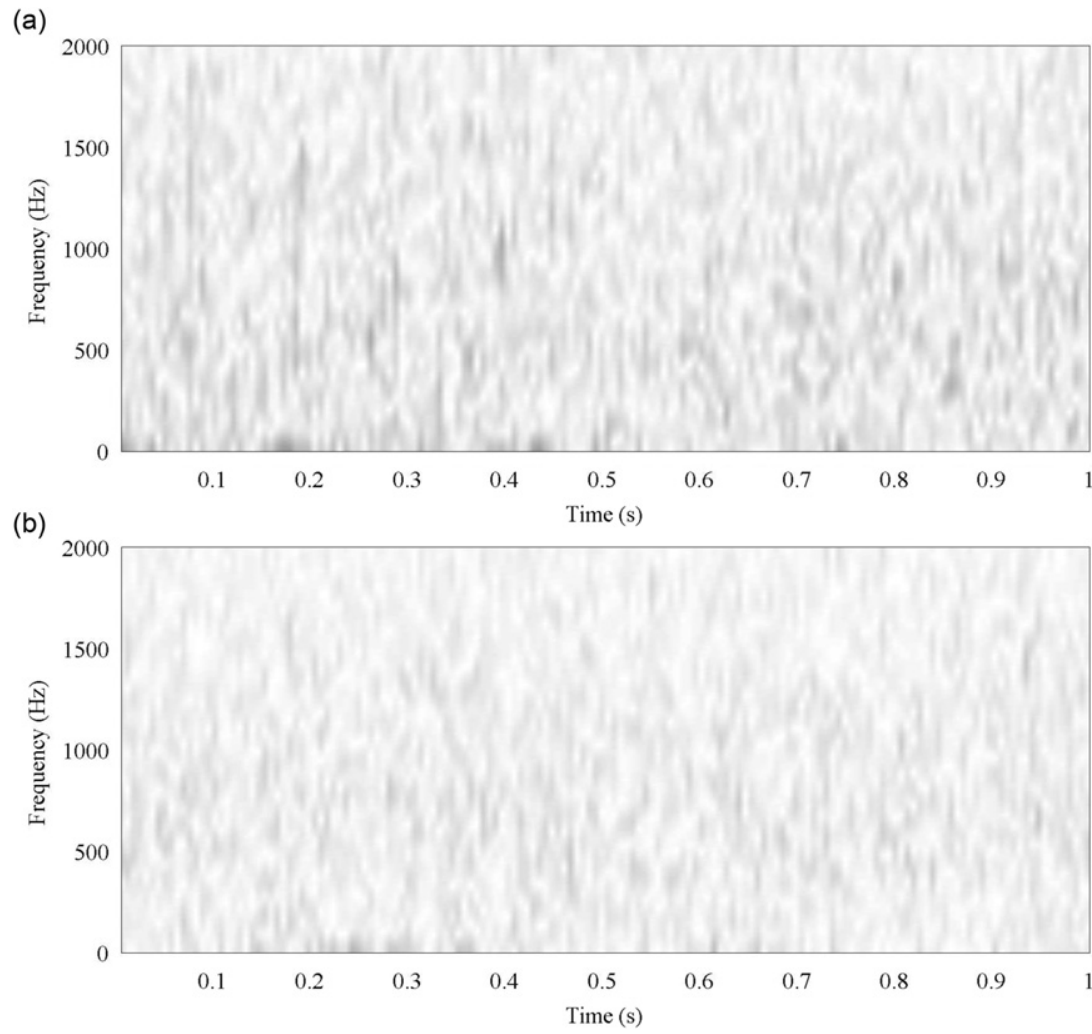

Fig. 13. Spectrograms of wall pressure fluctuations at $\alpha=6^{\circ}$ for two transducers along the suction side, $\operatorname{Re}=750000:(a) x / c=0.7$ and $(b) x / c=0.8$.

spectrum without any particular peak resulting from a fully turbulent boundary-layer flow conversely to the transition spectrum obtained for $\alpha=2^{\circ}$ and $4^{\circ}$.

\subsection{Strouhal number}

From the boundary-layer characteristics in Table 1, it is possible to compute the Strouhal number $\mathrm{St}_{\theta}$ of the LSB vortex shedding, defined from the vortex shedding frequency $f$, the momentum thickness at separation $(\theta)_{\text {sep }}$ and the external velocity at separation $\left(u_{\text {ext }}\right)_{\text {sep }}$, as $\mathrm{St}_{\theta}=f \times(\theta)_{\text {sep }} /\left(u_{\text {ext }}\right)_{\text {sep }}$. For a laminar separation occurring at $x / c=0.72$, using the frequency $f=395 \mathrm{~Hz}$ and the velocity $\left(u_{\text {ext }}\right)_{\text {sep }} \approx 5.8 \mathrm{~m} / \mathrm{s}$ determined through LDV measurements, we obtain a Strouhal number $\mathrm{St}_{\theta}=0.0076$, which is close to the value of $\mathrm{St}_{\theta}=0.0068$ found in Pauley et al. (1990). To take into account the uncertainty in the determination of the separation location, a mean Strouhal number may be computed from the average momentum thickness and external velocity between $x / c=0.68$ and $x / c=0.74$, which gives the value even closer of $\mathrm{St}_{\theta}=0.0074$. This confirms that the periodic pressure fluctuations observed in our experiments result from a vortex shedding process downstream of a Laminar Separation Bubble.

\subsection{Rigid hydrofoil vibration measurements}

Vibration measurements were performed for the rigid hydrofoil under the above-mentioned boundary-layer flows. The foil natural frequencies in water were determined previously using scan measurements and the first four frequencies were estimated as $f_{1}=115-120 \mathrm{~Hz}, f_{2}=410-430 \mathrm{~Hz}, f_{3}=1020 \mathrm{~Hz}$ and $f_{4}=1815 \mathrm{~Hz}$. Some vibration spectra also exhibit a lowamplitude peak around $f=250 \mathrm{~Hz}$, but the latter frequency seems rather associated with the fixation system.

The results of single-point vibration measurements are shown in Fig. 14 for $\alpha=2^{\circ}$ and $\alpha=4^{\circ}$. The first two natural frequencies are clearly observed at, respectively, $f_{1}=120 \mathrm{~Hz}$ and $f_{2}=420 \mathrm{~Hz}$. Both spectra present additional components below $f=420 \mathrm{~Hz}$. As discussed in the previous sections, such frequencies are related to the vortex shedding process induced by the Laminar Separation Bubble, whose dominant frequency was estimated about $400 \mathrm{~Hz}$. This is confirmed by comparing those spectra to the case of a fully turbulent boundary-layer flow (see Fig. 14 for $\alpha=8^{\circ}$ and a higher Reynolds number $\operatorname{Re}=1200000$ ), where such components are not visible. Whereas the amplitude of the first modal peak is subjected to few variations between the three cases presented in Fig. 14, it appears clearly that the laminar to turbulent transition amplifies the second vibration mode (pure torsion), with a maximum amplitude of about $2.16 \times 10^{-4} \mathrm{~m} / \mathrm{s}$. The amplitude of the third mode also appears increased by the transition, yet to a lesser extent. Due to the important 


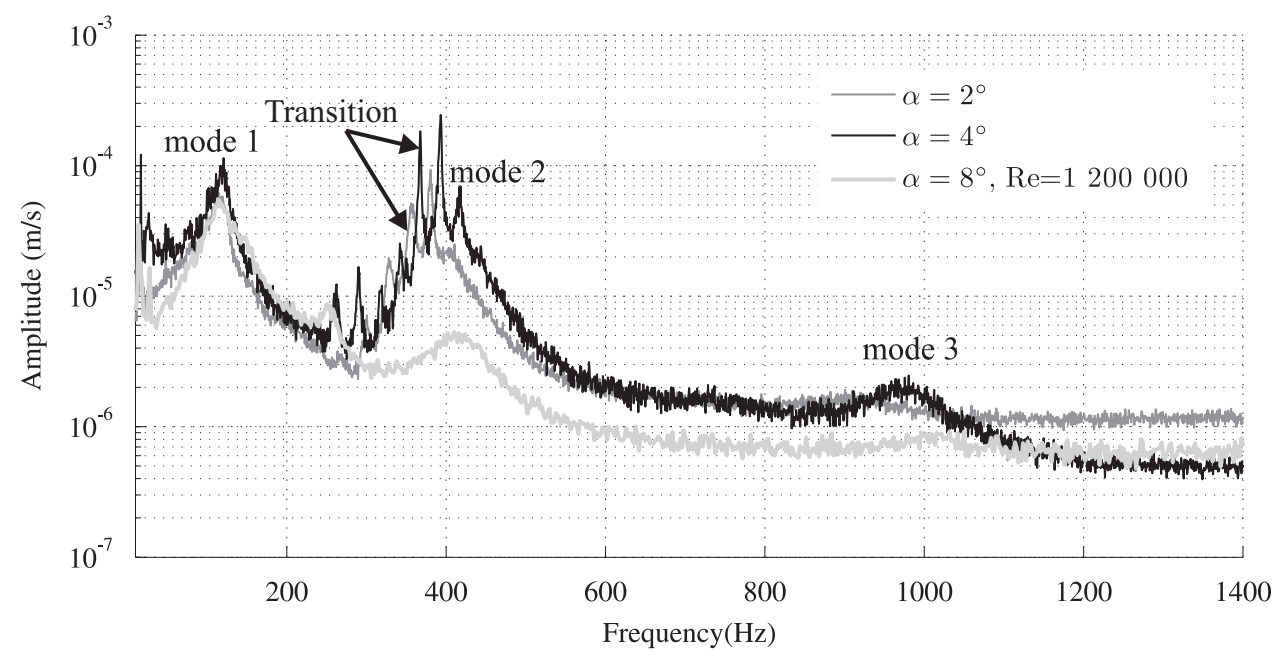

Fig. 14. Rigid hydrofoil, normal velocity spectra for $\alpha=2, \alpha=4^{\circ}$ at $\operatorname{Re}=750000$ and $\alpha=8^{\circ}$ at $\operatorname{Re}=1200000$.

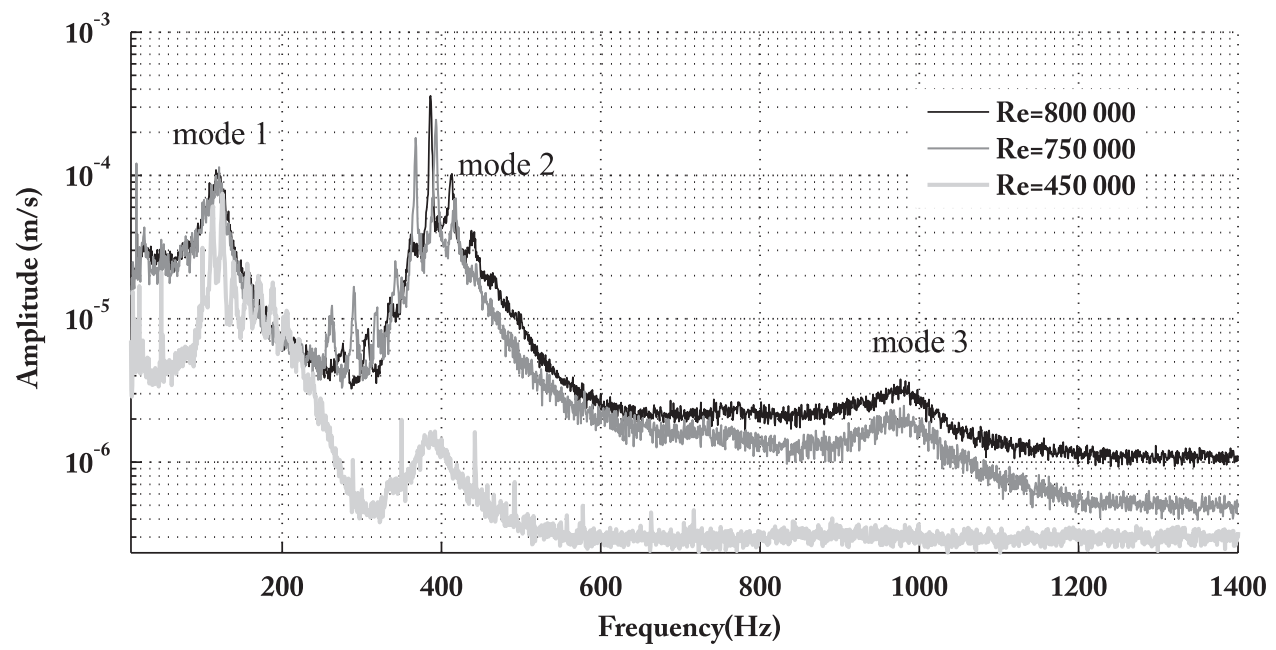

Fig. 15. Rigid hydrofoil, velocity spectra for $\alpha=4^{\circ}$ and various Reynolds numbers.

rigidity of the steel foil, the modes of higher natural frequencies remain unaffected by the transition and the growth of the second mode.

The Reynolds number effects are highlighted in Fig. 15 for an angle of incidence $\alpha=4^{\circ}$. The frequencies associated with the laminar to turbulent transition seem to increase with the Reynolds number, which is in agreement with the results of Pauley et al. (1990). At $\operatorname{Re}=450000$, the frequencies related to the transition lie below $f=200 \mathrm{~Hz}$ and only affect the first vibration mode of the hydrofoil. For that Reynolds number the global level of vibrations is relatively low, and the third mode is not excited. The vibration level is then seen to increase with the Reynolds number. At $R e=800000$ we observe a peak at relatively high amplitude $\left(3.5 \times 10^{-4} \mathrm{~m} / \mathrm{s}\right)$ at the second natural frequency. The latter mode seems to be near resonance. Moreover, the different peaks linked to the transition are increased of about $18 \mathrm{~Hz}$, as compared to $\operatorname{Re}=750000$. These observations lead to the conclusion that in the case of the rigid hydrofoil, the transition has a strong local effect on the foil vibrations but exhibit no interactions between the foil and the boundary-layer flow.

\subsection{Flexible hydrofoil vibration measurements}

In order to enhance the vibration level and the degree of interaction, a second hydrofoil made of a more flexible material has then been considered. As for the rigid hydrofoil, vibration measurements have been carried out for various angles of incidence and Reynolds numbers, and consist of both single-point measurements on a reflective patch located near the trailing edge (as mentioned previously, the low density of the material induced a weak reflecting level of the laser beam), and scanning measurements over the whole pressure side of the hydrofoil. For the latter, a grid of $9 \times 9$ 
measurement points in the longitudinal and spanwise directions and a frequency bandwidth of [0; 2] kHz have been chosen in order to recover an accurate description of the first modes of the hydrofoil. The visualization of the operating deflection shapes at the resonance frequencies observed on the vibration spectra enables the mode identification, as shown in Fig. 16 for the modes 2-4. The first four natural frequencies of the flexible hydrofoil in water have hence been determined as $f_{1}=43 \mathrm{~Hz}$ (first bending mode), $f_{2}=171 \mathrm{~Hz}$ (first torsional mode), $f_{3}=291 \mathrm{~Hz}$ (second bending mode) and $f_{4}=560 \mathrm{~Hz}$ (mixed bending and torsional mode).

Fig. 17 compares the spectra of the normal velocity of the hydrofoil measured on the reflective patch (observable in Fig. 16) for a Reynolds number of $\operatorname{Re}=800000$ and three angles of incidence $\alpha=2^{\circ}, \alpha=4^{\circ}$ and $\alpha=6^{\circ}$. As a consequence of the higher flexibility of this hydrofoil, a larger number of natural frequencies appears on the spectra. As for the rigid hydrofoil, the presence of a Laminar Separation Bubble is confirmed by comparing the spectra corresponding to the two extreme angle values: at $\alpha=6^{\circ}$, for which the boundary-layer flow is fully attached and turbulent from the leading edge, the vibration level is relatively low and the natural frequencies are the only distinctive frequencies; for $\alpha=2^{\circ}$, additional contributions related to the LSB vortex shedding appear between 400 and $600 \mathrm{~Hz}$ and lead to the amplification of the third and fourth modal peaks. The frequency values of these components will be discussed later on. At the higher incidence $\alpha=4^{\circ}$ and for the same Reynolds number $\mathrm{Re}=800$ 000, the Laminar Separation Bubble has similar characteristics than at $\alpha=2^{\circ}$, as already mentioned, and the vibration spectrum is hence very similar to the previous one, showing only slight amplitude variations around the fourth natural frequency (see Fig. 17).

Vibration measurements of the flexible hydrofoil at various Reynolds numbers show again that the freestream velocity can have on the contrary a strong impact on the LSB and the induced vibrations, as shown for instance in Fig. 18 for $\alpha=4^{\circ}$. As for the rigid hydrofoil, the amplitude of the additional components related to the LSB vortex shedding process is seen to increase with the Reynolds number, inducing a coherent growth of the surrounding modal peaks (apart from the first two modal peaks which remain similar whatever the Reynolds number). Fig. 18 also shows an increase of the frequencies

Mode 2

Mode 3

Mode 4

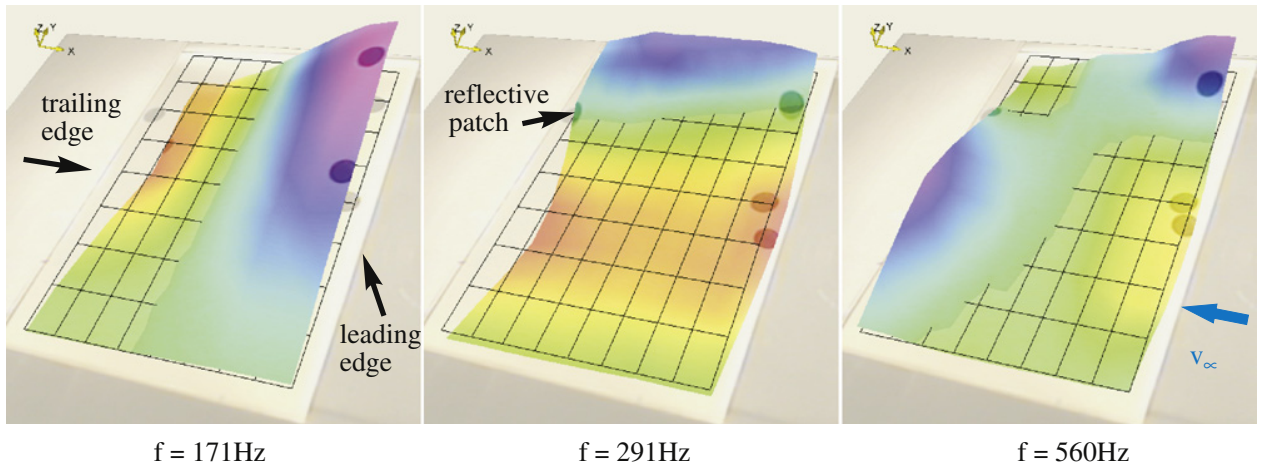

Fig. 16. Experimental mode shapes of the flexible hydrofoil obtained from scanning measurements at the grid points.

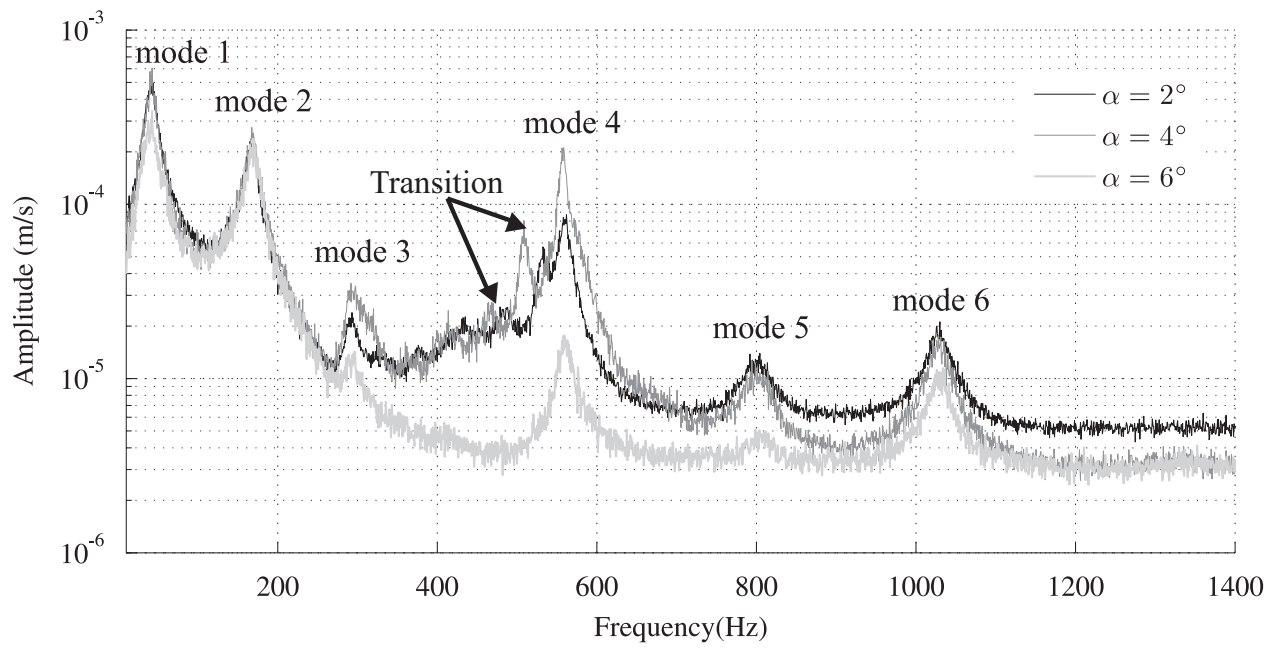

Fig. 17. Flexible hydrofoil normal velocity spectra for $\alpha=2^{\circ}, \alpha=4^{\circ}$ and $\alpha=6^{\circ}, \operatorname{Re}=800000$. 


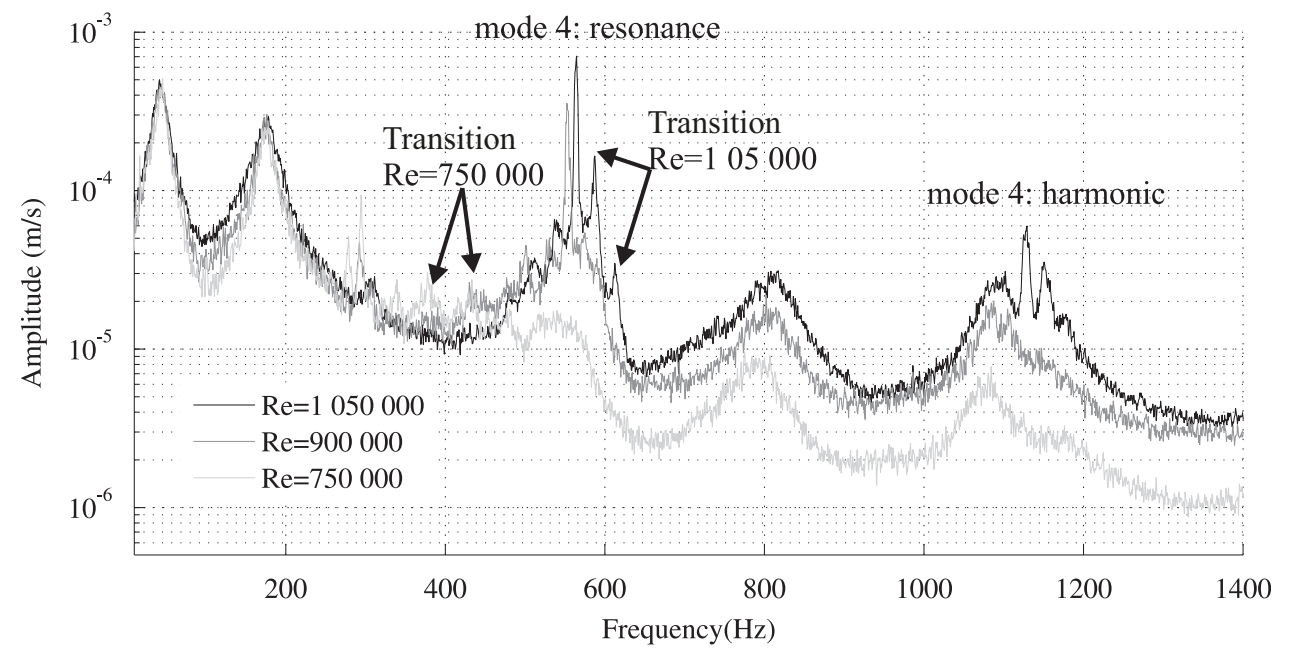

Fig. 18. Comparison of flexible hydrofoil velocity spectra for $\alpha=4^{\circ}$ and various Reynolds numbers.

associated with the transition process with the Reynolds number, and the interaction with the modal vibrations varies accordingly, as will be discussed afterwards.

The comparison between the vibrations measurements over the so-called rigid hydrofoil and the flexible one in the same configurations highlight discrepancies concerning the frequencies of the additional LSB-induced components, which seem increased in the presence of the flexible hydrofoil. The cases of an angle of incidence $\alpha=4^{\circ}$ with Reynolds numbers $\mathrm{Re}=750000$ and $\mathrm{Re}=800$ 000, for instance, illustrate the trends. At $\mathrm{Re}=750000$, the dominant vortex shedding frequency was estimated to be about $400 \mathrm{~Hz}$ from the analysis of pressure measurements over the rigid hydrofoil (Fig. 12). The vibration spectrum corresponding to the flexible hydrofoil clearly exhibits two additional peaks at 380 and $435 \mathrm{~Hz}$, respectively (Fig. 18, $\mathrm{Re}=750000$ ), whereas in the case of the rigid hydrofoil, the transition-induced frequencies remain below $400 \mathrm{~Hz}$ (Fig. 14 for $\alpha=4^{\circ}$ ). Similarly, the flexible hydrofoil vibration spectrum for $\operatorname{Re}=800000$ displays additional peaks above $500 \mathrm{~Hz}$ (Fig. 17) which are no more visible on the rigid hydrofoil corresponding spectrum (Fig. 15). These modifications may be due to the fluid-structure interaction between the hydrofoil vibrations and the boundary-layer flow. The LSB vortex shedding over the flexible hydrofoil may particularly be affected by the vibrations at the fourth natural frequency, which are particularly enhanced by the shedding excitation at close frequencies, as compared to the fully turbulent case.

It has to be noticed that the interaction intensity also depends on the Reynolds number and the involved vibration modes of the hydrofoil. As shown in Fig. 18, for $R e=750000$ the shedding-induced frequencies seem close to the third natural frequency of the hydrofoil. This results in a moderate enlargement of the corresponding modal peak, whose amplitude is naturally low as compared to the other modes (as shown in the fully turbulent configuration). For the higher Reynolds number $R e=1050000$, the amplification of the fourth modal peak reveals that the shedding frequency has now increased towards the fourth natural frequency and a resonance is observed. Here, the amplitudes of the modal peak and the surrounding additional components are such that harmonics are visible in the higher frequency domain, resulting again in a more complex interaction with the upper-ranked modes.

\section{Conclusion}

In this paper, the vibration of a hydrofoil submitted to a laminar to turbulent transition triggered by a Laminar Separation Bubble that may be observed in the boundary-layer along a hydrofoil, is studied experimentally. Flow velocity measurements have been used to determine, for given freestream velocity and angle of incidence, the localization and the size of the LSB on a steel hydrofoil referred to as the rigid hydrofoil. A vortex shedding process induced by the LSB has been identified from the measurement of periodic structures in wall-pressure fluctuations along the rigid hydrofoil. The vortex shedding frequency was characterized by a Strouhal number based on the boundary-layer characteristics at laminar separation of about 0.0076 . It was shown that the vortex shedding has a strong effect on the vibration of the hydrofoil by highly increasing the spectral response at the vortex shedding frequency. Particularly, it is shown that the frequencies associated with the vortex shedding of LSB are close to the second natural frequency and excite locally the corresponding mode. Far from the excitation frequency, the hydrofoil vibration level is globally very low and is not affected by the vortex shedding.

To emphasize the flow structure-interaction, the vibration of a flexible hydrofoil (with a lower Young modulus) was studied in the same operating conditions. The hydrofoil has the same geometry as the rigid one under zero loading and is mounted in a close configuration. The vortex shedding and the transition process are seen to enhance more significantly 
the vibrations of the hydrofoil, especially at structural natural frequencies close to that of the vortex shedding. Moreover, depending on their natural frequencies and mode shapes, vibration modes are likely to interact strongly with the boundary-layer flow along the hydrofoil. This is for example the case of the second torsional mode (mode 4) as compared to the second bending mode (mode 3). Due to this interaction, the frequencies associated with the vortex shedding and transition appear to be increased in the presence of the flexible hydrofoil, as compared to the rigid hydrofoil case. Finally, when the vortex shedding frequency gets closer to the fourth natural frequency of the flexible hydrofoil, a resonance is found, which produces a response with higher order modes resulting in a more complex fluid structure interaction process. Such resonance phenomena and induced flow modifications will be the subject of future works.

\section{References}

Alam, M., Sandham, N.D., 2000. Direct numerical simulation of short laminar separation bubbles with turbulent reattachment. Journal of Fluid Mechanics $410,1-28$.

Almutairi, J., Jones, L., Sandham, N., 2010. Intermittent bursting of a laminar separation bubble on an airfoil. AIAA journal 48 (2), $414-426$.

Ducoin, A., Astolfi, J.A., Deniset, F., 2009. Computational and experimental investigation of flow over a transient pitching hydrofoil. European Journal of Mechanics/B Fluids 28, 728-743.

Gaster, M., 1966. The structure and behaviour of laminar separation bubbles. AGARD CP 4, 813-854.

Gordnier, R., 2009. High fidelity computational simulation of a membrane wing airfoil. Journal of Fluids and Structures 25 (5), $897-917$.

Haggmark, C.P., Bakchinov, A.A., Alfredsson, P.H., 2000. Experiments on a two-dimensional laminar separation bubble. Philosophical Transactions: Mathematical, Physical and Engineering Sciences 358 (1777), 3193-3205.

Hain, R., Kähler, C., Radespiel, R., 2009. Dynamics of laminar separation bubbles at low-Reynolds-number aerofoils. Journal of Fluid Mechanics 630, 129-153.

Jones, L.E., Sandberg, R.D., Sandham, N.D., 2008. Direct numerical simulations of forced and unforced separation bubbles on an airfoil at incidence. Journal of Fluid Mechanics 602, 175.

Leroux, J.B., Coutier-Delgosha, O., Astolfi, J.A., 2005. A joint experimental and numerical study of mechanisms associated to instability of partial cavitation on two-dimensional hydrofoil. Physics of Fluids 17, 052101

Lian, Y., Shyy, W., 2007. Laminar-turbulent transition of a low Reynolds number rigid or flexible airfoil. AIAA Journal 45 (7), $1501-1513$.

Marquillie, M., Ehrenstein, U., 2003. On the onset of nonlinear oscillations in a separating boundary-layer flow. Journal of Fluid Mechanics 490, 169-188.

Pauley, L.L., Moin, P., Reynolds, W.C., 1990. The structure of two-dimensional separation. Journal of Fluid Mechanics $220,397-411$.

Poirel, D., Harris, Y., Benaissa, A., 2008. Self-sustained aeroelastic oscillations of a NACA0012 airfoil at low-to-moderate Reynolds numbers. Journal of Fluids and Structures 24 (5), 700-719.

Poirel, D., Yuan, W., 2010. Aerodynamics of laminar separation flutter at a transitional Reynolds number. Journal of Fluids and Structures 26 (7-8), 1174-1194.

Richez, F., Mary, I., Gleize, V., Basdevant, C., 2008. Near stall simulation of the flow around an airfoil using zonal RANS/LES coupling method. Computers and Fluids 37 (7), 857-866.

Rist, U., Maucher, U., 2002. Investigations of time-growing instabilities in laminar separation bubbles. European Journal of Mechanics/B Fluids 21 (5), 495-509.

Tani, I., 1964. Low-speed flows involving bubble separations. Progress in Aerospace Sciences 5, 70-103.

Visbal, M.R., Gordnier, R.E., Galbraith, M.C., 2009. High-fidelity simulations of moving and flexible airfoils at low Reynolds numbers. Experiments in Fluids 46, 903-922.

Yang, Z., Voke, P.R., 2001. Large-eddy simulation of boundary-layer separation and transition at a change of surface curvature. Journal of Fluid Mechanics $439,305-333$. 